\title{
TÉTANO EN Cebus capucinus EN CAUTIVERIO: REPORTE DE CASO
}

\section{TETANUS IN CAPTIVE Cebus capucinus: CASE REPORT}

\author{
Teresa Oviedo-Socarrás ${ }^{1}$, José Cardona-Ávarez ${ }^{2}$, Misael Oviedo-Pastrana $^{3}$
}

\begin{abstract}
${ }^{1}$ MVZ, M.Sc, Ph.D. Universidad de Córdoba, Facultad de Medicina Veterinaria y Zootecnia, Departamento de Ciencias Pecuarias. Carrera 6 No. 76-103, Km. 3, vía Cereté, Córdoba-Colombia, e-mail: toviedo@correo.unicordoba.edu.co; ${ }^{2}$ MVZ, MSc, Ph.D. Universidad de Córdoba, Facultad de Medicina Veterinaria y Zootecnia, Departamento de Ciencias Pecuarias. Carrera 6 No. 76-103, Km. 3, vía Cereté, Córdoba-Colombia, e-mail: cardonalvarez@hotmail.com; ${ }^{3}$ MVZ, MSc, Ph.D. Universidad de Córdoba, Instituto de Investigaciones Biológicas del Trópico. Carrera 6 No. 76-103, Km. 3, vía Cereté, Córdoba-Colombia, e-mail: mpastrana@outlook.com.
\end{abstract}

Rev. U.D.C.A Act. \& Div. Cient. 19(2): 489-492, Julio-Diciembre 2016

\section{INTRODUCCIÓN}

Aunque en la región no existe un estudio metódico sobre el número de primates en cautiverio es bien sabido que varias de estas especies son víctimas del comercio ilegal, entre ellas, el Cebus capucinus, conocido también como maicero capuchino, mono capuchino o mono carablanca. Según Almirón et al. (2005), un número considerable de estos primates acaban incautados para posterior rehabilitación en centros de rescate, como resultado de esfuerzos gubernamentales por controlar el tráfico ilegal de fauna silvestre; sin embargo, muchos son tenidos ilegalmente como mascotas, en condiciones inapropiadas, de acuerdo a lo reportado por la International Primatological Society (2007), lo que favorece la presentación de graves patologías, entre las cuales, podría estar el tétano. Adicionalmente, Karesh et al. (2005) enfatizan que el comercio ilegal de vida silvestre es una forma de dispersión de enfermedades infectocontagiosas, con diseminación rápida zoonótica o antropozoonótica, entre las poblaciones de animales domésticos y silvestres.

El tétano es una grave enfermedad neurológica causada por la neurotoxina del Clostridium tetani, una bacteria anaerobia Gram positiva, formadora de esporas, capaz de sobrevivir largos periodos de exposición al aire y otras condiciones ambientales adversas. Esta bacteria está ampliamente distribuida y tiene su hábitat natural en el suelo; sin embargo, puede ser aislada de heces de animales domésticos y de seres humanos (Afshar et al. 2011).

Generalmente, la infección con el agente etiológico se produce a través de la contaminación de heridas sucias, profun- das y con tejido necrótico. Ante las condiciones anaerobias de la herida, el C. tetani secreta dos toxinas, conocidas como tetanolisina y tetanospasmina. El papel de la tetanolisina no está totalmente desvendado en el tétanos, mientras que la tetanospasmina es la neurotoxina responsable por el síndrome clínico, característico del tétano (Bleck \& Brauner, 2004).

El presente reporte tuvo como objetivo relatar un caso de tétano en un primate Cebus capucinus en cuativerio y, al mismo tiempo, alertar sobre la gravedad de la tenencia de animales silvestres.

\section{MATERIALES Y MÉTODOS}

Fue llevado a la sala de necropsia del área de patología animal de la Facultad de Medicina Veterinaria y Zootecnia de la Universidad de Córdoba, Colombia, un primate adulto, macho entero, de la especie $C$. capucinus. Se informó, que el animal, tenido como mascota, permanecía amarrado con cadena metálica a nivel de la cintura y previo a la muerte presentó un cuadro clínico caracterizado por contracción severa, con la cabeza curvada hacia atrás y espasmos musculares generalizados que, finalmente, lo llevaron a óbito.

Durante la inspección externa, se observó un estado de rigidez cadavérica generalizado; sin embargo, llamó la atención la posición del animal, que presentaba la cabeza, la espalda y las extremidades traseras totalmente curvadas hacía atrás, la cola bien estirada e igualmente curvada hacia atrás y las extremidades anteriores cruzadas al frente del cuerpo con las muñecas dobladas (Figura 1A y 1B). 

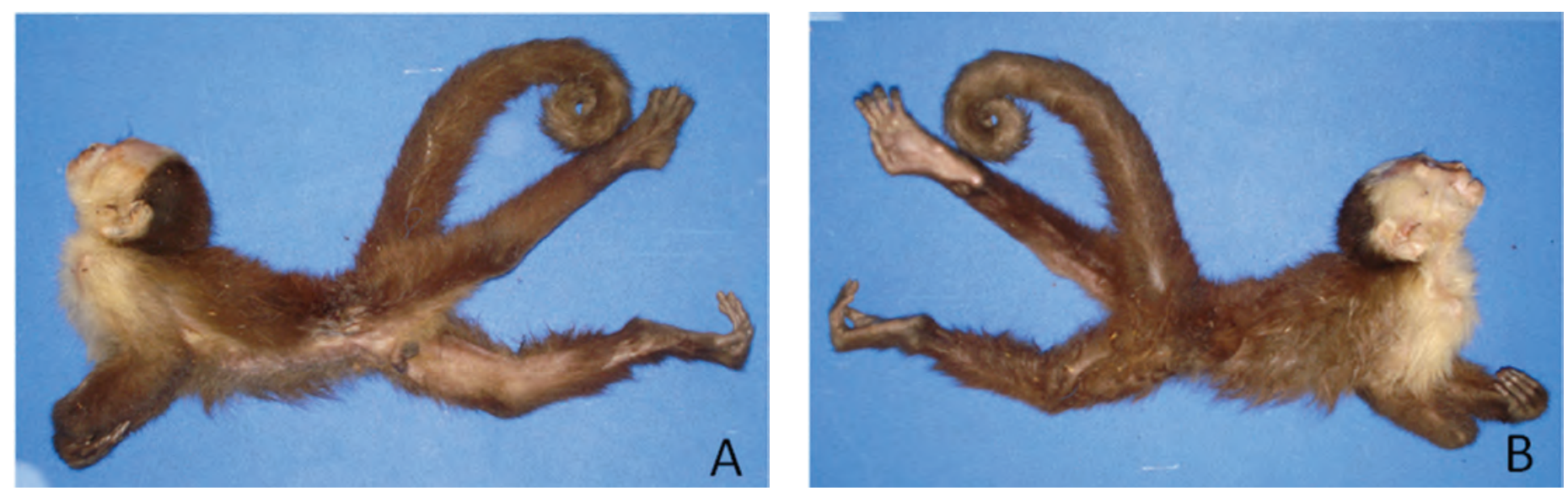

Figura 1. Opistótonos, con postura rígida severa, observado como signo clínico característico de tétanos, en un ejemplar de Cebus capucinus macho. A) Vista lateral izquierda y B) Vista lateral derecha.

Al inspeccionar detalladamente la piel, se evidenció una herida extensa en el lado izquierdo del abdomen, con pelos adheridos a la misma. Próximo a la herida, se observaron áreas hiperpigmentadas de lesiones previas, plenamente cicatrizadas. Al retirar los pelos, se evidenció la herida ulcerada y con abundante tejido necrótico (Figura 2A y 2B).
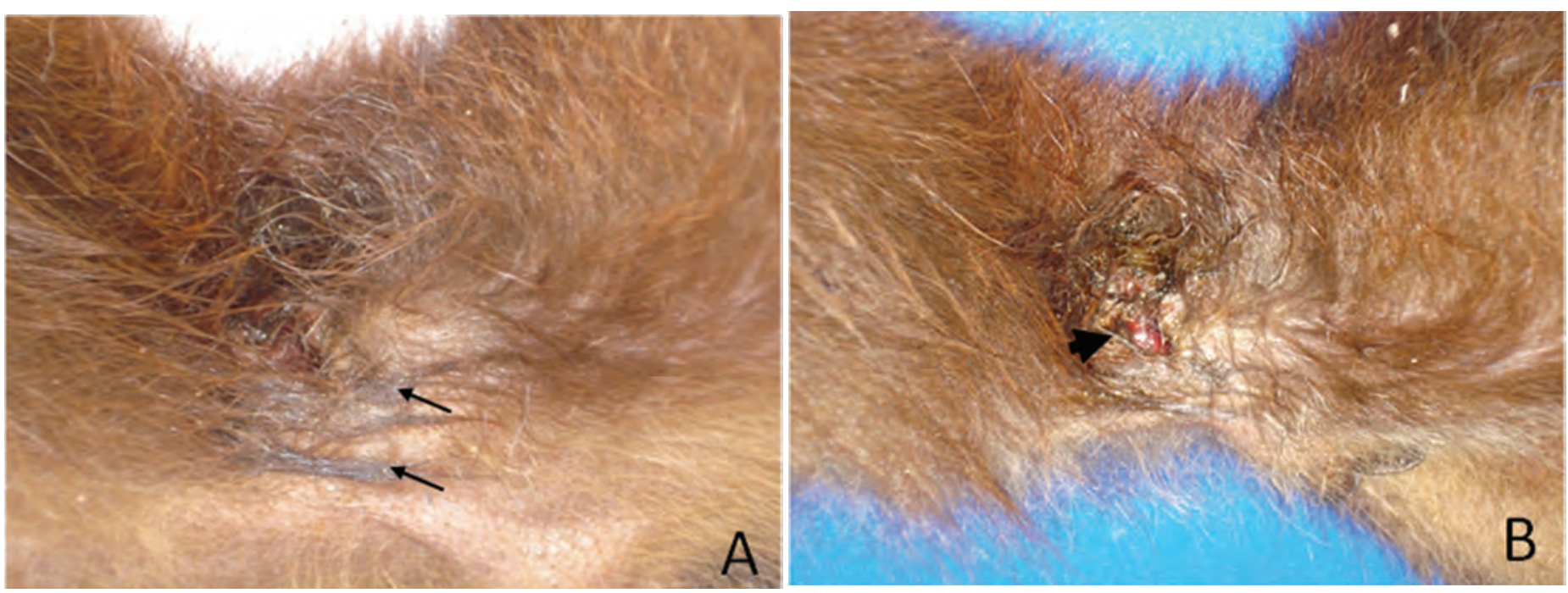

Figura 2. Detalle de herida, a nivel abdominal en Cebus capucinus. A) Herida con pelos adheridos (flechas); se observan claramente áreas hiperpigmentadas, de lesiones previas cicatrizadas, producidas por el sistema de sujeción utilizado en el animal (flechas) y B) Herida ulcerada (cabeza de flecha), con presencia de abundante tejido necrótico.

Durante la inspección interna del animal, no fueron observadas lesiones relevantes, ni alteraciones en los aparatos respiratorio y gastrointestinal, sugestivas de envenenamiento.

\section{RESULTADOS Y DISCUSIÓN}

El caso fue diagnosticado como tétano, debido a los signos clínicos que presentó el animal, previo a la muerte, con contracturas musculares y opistótonos, característicos de la enfermedad, confirmados a la necropsia, así como la presencia 
de herida necrótica, con condiciones de anaerobiosis, ideales para la penetración y el crecimiento del $C$. tetani, aunque no fue realizado aislamiento del agente etiológico de la lesión, ni confirmación de la toxina por pruebas de toxicidad.

La tenencia ilegal de primates, casi siempre va de la mano con la falta de infraestructura adecuada para estas especies, que obliga a los propietarios a tomar medidas inapropiadas para evitar que escapen y, la más común, es amarrar a los animales por la cintura, con correas o cadenas metálicas. Este sistema de sujeción puede lesionar gravemente la piel de los animales, ya que, por naturaleza, tienen muchos desplazamientos y el roce repetitivo de las correas con la piel, puede causar heridas serias, que se convierten en focos de entrada para agentes bacterianos.

Exámenes de laboratorio difícilmente proporcionan un diagnóstico definitivo de tétano y el agente etiológico, raramente, se recupera del sitio de la infección y no hay respuesta de anticuerpos detectable, incluso, en los casos graves, por lo tanto, los médicos se deben basar en resultados clínicos para el diagnóstico rápido. Según la OMS, el tétano en adultos, incluye, trismo o risa sardónica y contracciones musculares dolorosas, con antecedentes de infección de una herida (Kessler et al. 2015).

Basados en los antecedentes ocurridos antes de la muerte del animal, como contracción severa, cabeza curvada hacia atrás y espasmos musculares generalizados, asociado a los hallazgos descritos durante la necropsia, como opistótonos y lesión en piel con condiciones adecuadas para el crecimiento de $C$. tetani, se diagnosticó el caso como tétano, según método descrito como mecanismo de diagnóstico clínico de la enfermedad (Schiavo et al. 1992; Chen et al. 2008; Armijo et al. 2012). Esta enfermedad ha sido reportada en primates no humanos en diversas partes del mundo, como es el caso de Macaca mulatta (Digiacomo \& Missakian, 1972; Rawlins \& Kessler, 1982; Springer et al. 2009), Saimiri sciureus (Kessler \& Brown, 1979), Papio cynocephalus Anubis (Goodwin et al. 1987) y Macaca fuscata (Nakano et al. 2012); sin embargo, no se encontraron relatos en la literatura consultada en la especie Cebus capucinus.

El animal convivía en un hogar del municipio de Montería, Córdoba, que posee condiciones agroecológicas, como humedad relativa del $82 \%$ y temperatura promedio de $28^{\circ} \mathrm{C}$, que favorece el crecimiento y la replicación del microorganismo.

La puerta de entrada del $C$. tetani suele ser a través de heridas sucias y profundas, donde el tejido necrosado e infectado proporciona un ambiente anaeróbico apropiado para la germinación de las esporas, con la posterior elaboración y secreción de toxinas, por el agente etiológico (Cook et al.
2001), situación que fue observada en el espécimen necropsiado.

Las principales toxinas producidas por C. tetani son tetanolisina, una toxina hemolítica, cuyo significado biológico no ha sido del todo aclarado; sin embargo, ha sido relacionada con la invasión bacteriana, a nivel local en el tejido y tetanospasmina, la neurotoxina responsable por las manifestaciones clínicas de esta patología y que fueron observadas en este animal. Tras la producción y liberación en la herida, la tetanospasmina se difunde y se une a los gangliósidos $\mathrm{GD}_{1 \mathrm{~b}}$ y $\mathrm{GT}_{1 \mathrm{~b}}$ de los nervios periféricos.

La toxina es posteriormente internalizada y transportada retrógradamente intra-axón hasta el cuerpo celular de las neuronas inhibidoras de la médula espinal, donde bloquea la liberación de neurotransmisores inhibitorios, como el ácido gamma-aminobutírico y glicina. Esas sustancias son inhibidoras de la neurotransmisión, por lo tanto, su ausencia lleva a la parálisis espástica, característica del cuadro clínico de tétanos (Chen et al. 2008).

Cuatro manifestaciones clínicas de tétanos han sido relatadas en la literatura: generalizada, localizada, cefálica y neonatal. La forma generalizada, observada en el presente reporte de caso, es la más común y severa y se manifiesta con signos de hiperactividad autonómica. Entre los signos más frecuentes, se encuentra el trismo, la rigidez cervical, la risa sardónica, el abdomen en tabla, períodos de apnea por contracciones de músculos torácicos, disfagia y opistótonos (Armijo et al. 2012). Aunque el opistótonos se puede presentar en diversas situaciones clínicas (RIES et al. 1994) es, en general, reconocido como un signo específico del tétanos, cuando es asociado a las demás condiciones de la enfermedad.

Ante este reporte de caso, se hace necesario señalar que la extracción de fauna silvestre de su hábitat natural, para ser usados como mascotas, tiene implicaciones negativas en el bienestar y en la conservación de las especies. Los métodos de captura, de transporte y de tenencia de estos animales causan, en su mayoría, muerte por estrés y enfermedades, como la aquí descrita, sin desconsiderar el riesgo para la salud humana, ya que las enfermedades emergentes y reemergentes, originadas en animales de vida silvestre, pueden ser transmitidas a poblaciones humanas, por contacto directo o por vectores, determinando así una gran problemática social y epidemiológica.

Conflicto de intereses: El manuscrito fue preparado y revisado por todos los autores, quienes declaramos que no existe conflicto de intereses que ponga en riesgo la validez de los resultados presentados. 


\section{BIBILIOGRAFÍA}

1. AFSHAR, M.; RAJU, M.; ANSELL, D.; BLECK, T.P. 2011. Narrative review: tetanus a health threat after natural disasters in developing countries. Ann. Intern. Med. (Estados Unidos). 54(5):329-335.

2. ALMIRÓN, M.; FLORES, C.N.; GONZALEZ, S.T.; HORRISBERGER, H.S. 2005. El tétanos. Rev. Posgrado de la Vla Cátedra de Medicina. (Argentina). 143:2227.

3. ARMIJO, M.J.; SOTO-AGUILAR, F.; BRITO, C. 2012. Tétanos generalizado: caso clínico y revisión del tema Generalized tetanus: clinical case report and review of the literature. Rev. Chil. Neuro-Psiquiat. 50(4):229233.

4. BLECK, T.P.; BRAUNER, J.S. 2004. Tetanus. In: Scheld, W.M.; Whitley, R.J.; Marra, C.M. (eds.) Infections of the central nervous system. 3rd ed. New York: Lippincott Williams \& Wilkins. p.625-648.

5. CHEN, C.; BALDWIN, M.R.; BARBIERI, J.T. 2008. Molecular basis for tetanus toxin coreceptor interactions. Biochemistry. (Estados Unidos). 47(27):7179-7186.

6. COOK, T.M.; PROTHEROE, R.T.; HANDEL. J.M. 2001. Tetanus: a review of the literature. Br. J. Anaesth. 87:477-487.

7. DIGIACOMO, R.F.; MISSAKIAN, E.A. 1972. Tetanus in a free-ranging colony of Macaca mulatta: a clinical and epizootiologic study. Lab. Anim. Sci. 22:378-83.

8. GOODWIN, W.J.; HAINES, R.J.; BERNAL, J.C. 1987. Tetanus in baboons of a corral breeding colony. Lab. Anim. Sci. 37:231-232.

9. INTERNATIONAL PRIMATOLOGICAL SOCIETY. 2007. Comité de manejo en cautividad de la Sociedad Internacional de Primatología. Directrices Internacionales para la adquisición, cuidado y reproducción de primates no humanos. Disponible desde internet en: http://www.internationalprimatologicalsociety.org (con acceso 22/04/2015).
10. KARESH, W.B.; COOK, R.A.; BENNETT, E.L.; NEWCOMB, J. 2005. Wildlife trade and global disease emergence. Emerg. Infect. Dis. 11(7):1000-1002.

11. KESSLER, M.J.; BROWN, R.J. 1979. Clinical description of tetanus in squirrel monkeys (Saimiri sciureus). Lab. Anim. Sci. 29(2):240-242.

12. KESSLER, M.J.; HERNÁNDEZ, P.R.; RAWLINS, R.G.; RUIZ-LAMBRIDES, A.; DELGADO, D.L.; SABAT, A.M. 2015. Long-term effects of tetanus toxoid inoculation on the demography and life expectancy of the Cayo Santiago rhesus macaques. Am J Primatol.. 77(2):211-221.

13. NAKANO, T.; NAKAMURA, S.; YAMAMOTO, A.; TAKAHASHI, M.; UNE, Y. 2012. Tetanus as cause of mass die-off of captive Japanese macaques, Japan, 2008. Emerg Infect Dis. 18(10):1633-1635.

14. RAWLINS, R.G.; KESSLER, M.J. 1982. A five-year study of tetanus in the Cayo Santiago rhesus monkey colony: behavioral description and epizootiology. Am. J Primatol. 3:23-39.

15. RIES, C.R.; SCOATES, P.J.; PUIL, E. 1994. Opisthotonos following propofol: a nonepileptic perspective and treatment strategy. Can J Anaesth. 41:414-419.

16. SCHIAVO, G.; BENFENATI, F.; POULAIN, B.; ROSSETTO, O.; POLVERINO, P.; DASGUPTA, BR.; MONTECUCCO, C. 1992. Tetanus and botulinum-B neurotoxins block neurotransmitter release by proteolytic cleavage of synaptobrevin. Nature. 359(6398):832835.

17. SPRINGER, D.A.; PHILLIPPI-FALKENSTEIN, K.; SMITH, G. 2009. Retrospective analysis of wound characteristics and tetanus development in captive macaques. J. Zoo Wildl. Med. (Estados Unidos). 40:95102.

Recibido: Mayo 14 de 2016

Aceptado: Octubre 10 de 2016

Cómo citar:

Oviedo-Socarrás, T.; Cardona-Àvarez, J.; Oviedo-Pastrana, M. 2016. Tétano en Cebus capucinus en cautiverio: reporte de caso. Rev. U.D.C.A Act. \& Div. Cient. 19(2): 489-492. 